\title{
Ensayo Pictórico: Descripción del posicionamiento del túnel femoral en distintas técnicas de reconstrucción del ligamento cruzado anterior (Ica)
}

Marcelo San Martín Montes ${ }^{2}$, Mario Mora Valdés ${ }^{2}$, Jorge Llanos Cáceres ${ }^{1}$, Nicolás Zilleruelo Vásquez ${ }^{1}$, Erika Morales López', Gonzalo Serrano Belmar'.

1. Médico Radiólogo. Clínica Alemana. Santiago - Chile.

2. Fellow MSK. Clínica Alemana. Santiago - Chile.

Pictory Essay: Description of the positioning of the femoral tunnel in different techniques of reconstruction of the anterior cruciate ligament

\begin{abstract}
In daily radiological practice, we frequently find different ACL reconstruction techniques, which we must know in order to achieve adequate interpretation of the findings.

In this pictorial essay, we show different types of LCA reconstruction techniques, highlighting the anatomical reconstruction concept and presenting different positions of the femoral tunnel, seeking to deliver tools to the radiologist in order to recognize the normal postoperative findings and possible complications. Keywords: LCA reconstruction, femoral tunnel, postoperative.
\end{abstract}

Resumen. En la práctica radiológica habitual el radiólogo se encuentra frecuentemente con distintas técnicas de reconstrucción del LCA que debe conocer de manera general para una adecuada descripción e interpretación de los hallazgos.

En el presente ensayo pictórico exponemos diferentes tipos de técnica de reconstrucción del LCA, con énfasis en el concepto de reconstrucción anatómica, presentando distintas posiciones del túnel femoral, buscando entregar herramientas al radiólogo para reconocer la normalidad postoperatoria e interpretar posibles complicaciones.

Palabras clave: reconstrucción LCA, túnel demoral, postoperatorio.

San Martín M, et al. Ensayo Pictórico: Descripción del posicionamiento del túnel femoral en distintas técnicas de reconstrucción del ligamento cruzado anterior (Ica). Rev Chil Radiol 2018; 24(2): 63-66.

Correspondencia: Marcelo San Martín / msanmartinmontes@gmail.com

Trabajo enviado el 23 de julio de 2018. Aceptado para publicación el 07 de agosto de 2018.

\section{Introducción}

En nuestra práctica diaria nos encontramos con distintas técnicas de posicionamiento de los túneles tibial y femoral en la cirugía de reconstrucción del LCA.

La variabilidad de estas técnicas y los hallazgos imaginológicos asociados, presentan un desafío que cada radiólogo debe enfrentar.

\section{Objetivos}

- Exponer diferentes tipos de técnica de reconstrucción del LCA, con énfasis en el concepto de reconstrucción anatómica.

- Presentar las distintas posiciones en los túneles femorales.

- Entregar herramientas al radiólogo para reco- nocer la normalidad post operatoria.

\section{Desarrollo del Tema}

La reconstrucción del LCA constituye una de las cirugías traumatológicas más frecuentes. Desde el inicio de las técnicas quirúrgicas, se ha planteado el concepto de reconstrucción anatómica, descrito como la recuperación funcional del LCA a sus dimensiones, orientación y sitios de inserción originales. El injerto de banda única con posicionamiento transtibial ha sido el método más ampliamente usado en la cirugía de LCA. Algunos estudios han demostrado permanencia de los síntomas de inestabilidad de tipo rotacional, lo que ha planteado el uso de otras técnicas (Figura 1). 
El abordaje artoscópico con portal accesorio anteromedial permitiría una reconstrucción más anatómica, intentando a través de una orientación más oblicua en el eje coronal, lograr un origen más cercano a la huella de inserción original del LCA (foot print). Los hallazgos radiológicos son acordes con este concepto y NO deben confundirse con hallazgos patológicos (Figura 2).
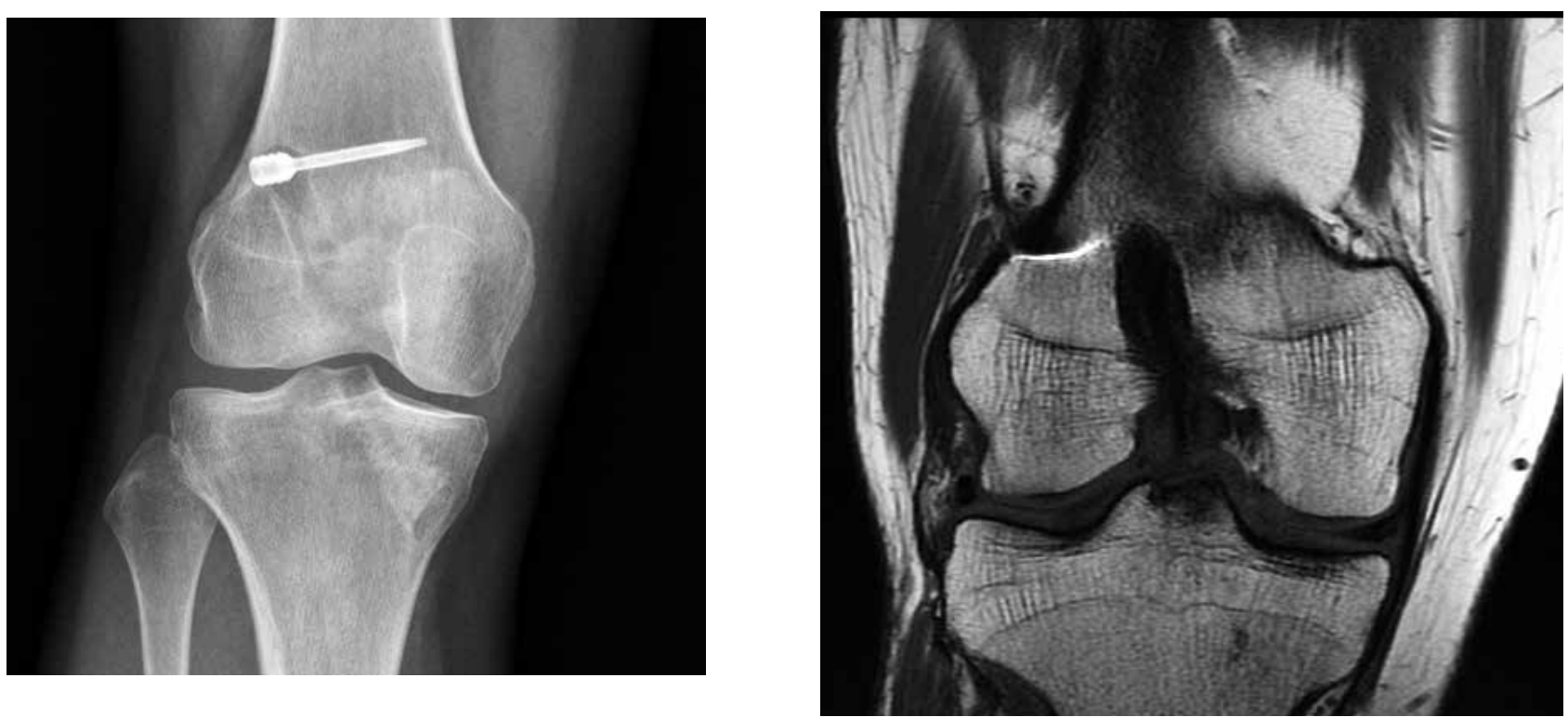

Figura 1. Radiografía frontal y resonancia magnética (RM) coronal ponderada en T1 de rodilla derecha que muestra una reconstrucción con técnica clásica transtibial. Destaca la inclinación del túnel femoral en el eje coronal, que se acerca al plano longitudinal
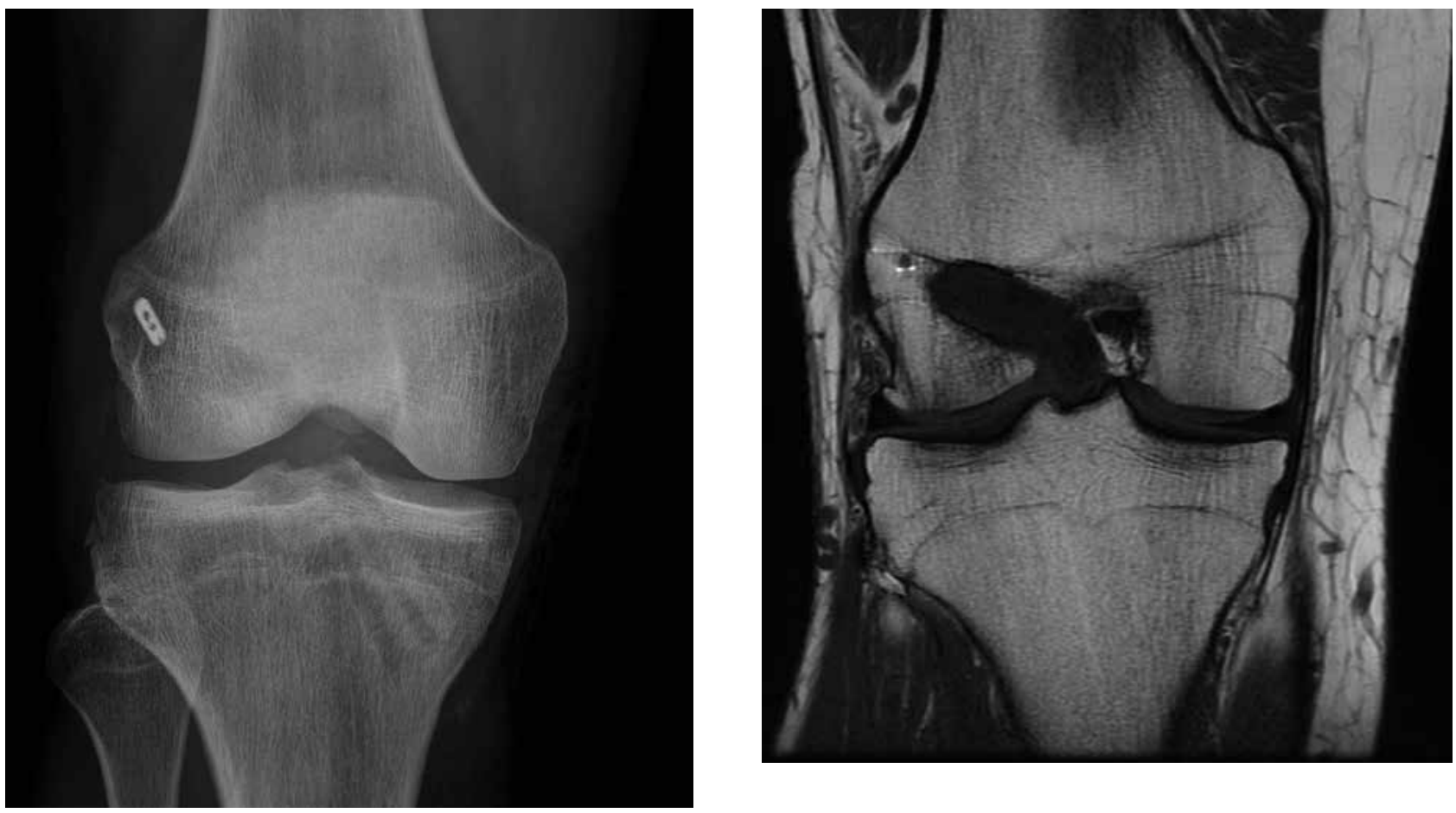

Figura 2. Radiografía frontal y RM coronal ponderada en T1 de rodilla derecha que muestra una reconstrucción con portal accesorio antero medial. En este caso la inclinación del túnel femoral en el eje coronal es más oblicua, acercándose al plano horizontal buscando la huella de inserción nativa para una reconstrucción anatómica. 
En el caso de pacientes adolescentes que requieren reconstrucción del LCA, y que aún mantienen fisis funcionales, se hace necesario una inclinación aún mayor del túnel femoral, respetando la fisis, hallazgo esperable en los controles radiológicos sucesivos (Figura 3).

En búsqueda del concepto de reconstrucción

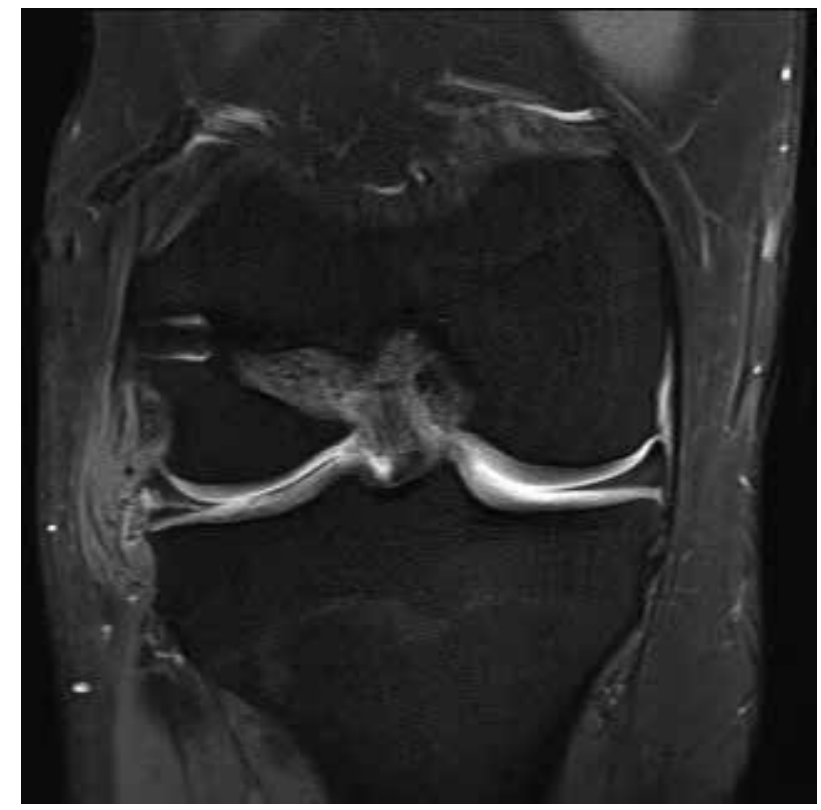

anatómica, existen grupos que buscan la reconstitución de las 2 bandas que conforman el LCA nativo. El control radiológico de estos pacientes es acorde con esta técnica, observando dos túneles tibiales y femorales, así como 2 bandas de injerto, lo que NO debe confundirse con una segunda reconstrucción por rotura de la primera plastía (Figura 4).

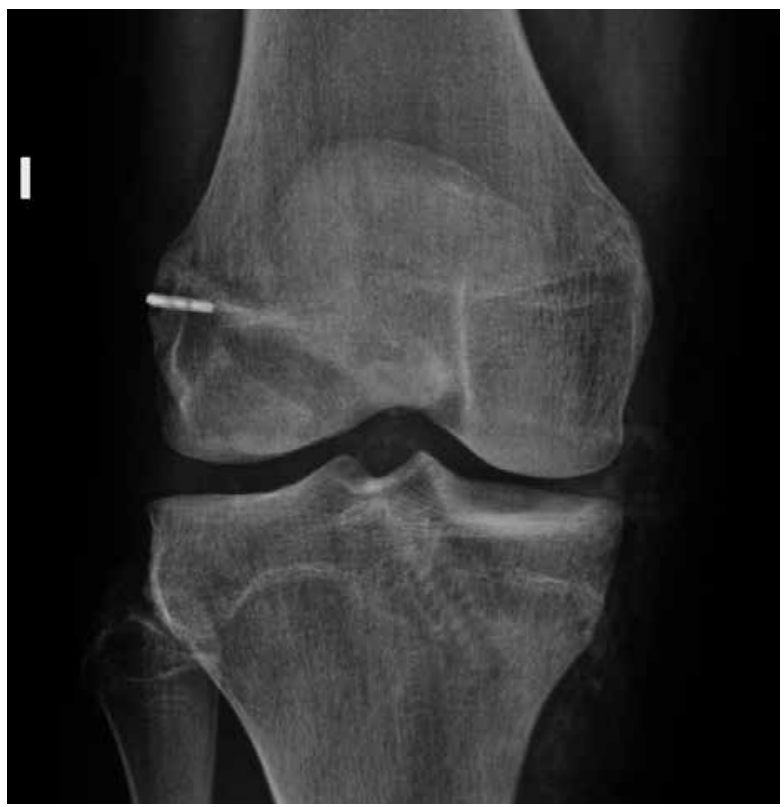

Figura 3. Radiografía frontal y RM coronal ponderada en DPFS de rodilla izquierda que muestra una reconstrucción con túnel femoral epifisiario. Esta técnica se utiliza en pacientes con fisis abierta, buscando disminuir su daño y compromiso del crecimiento óseo.
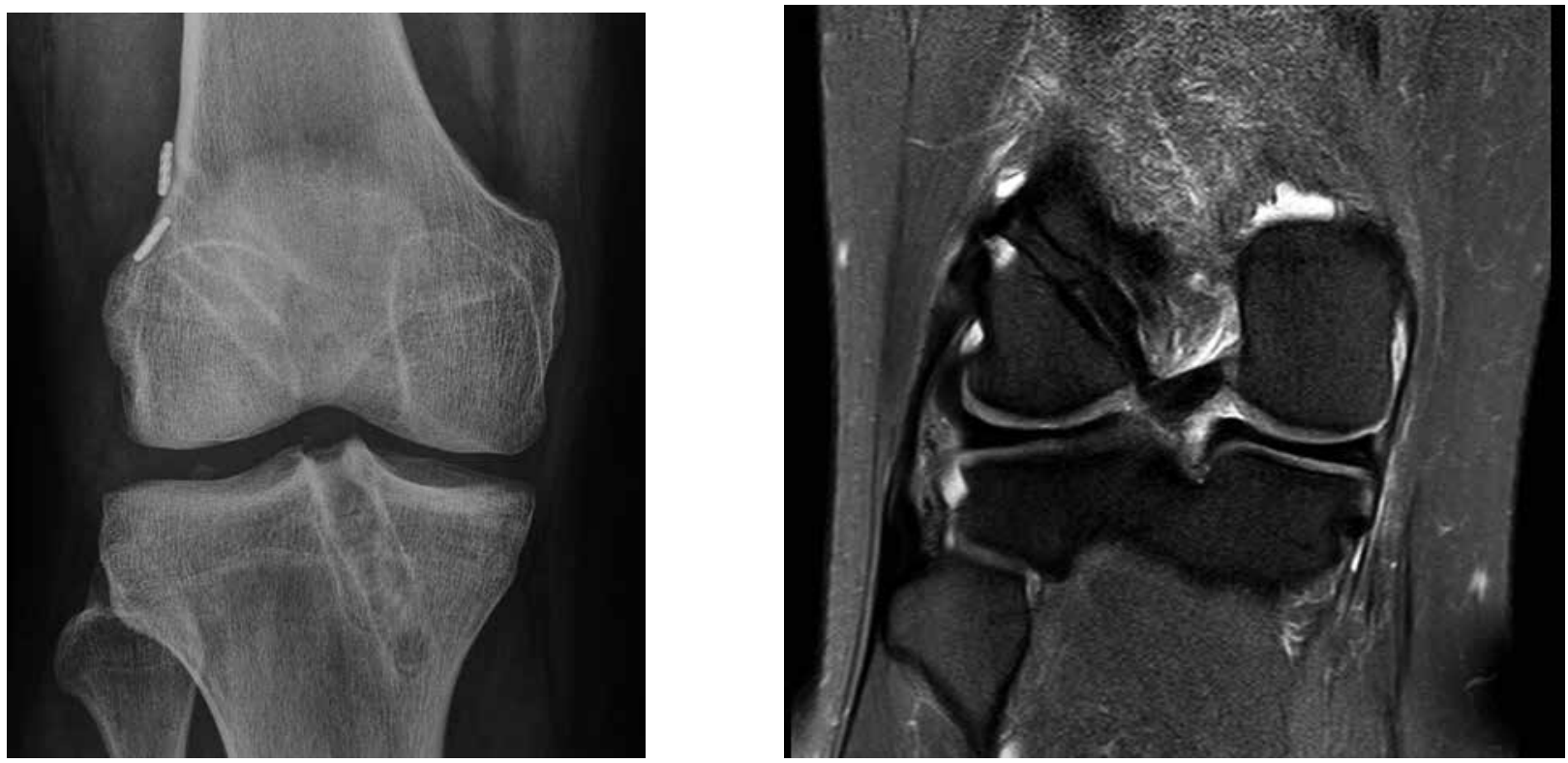

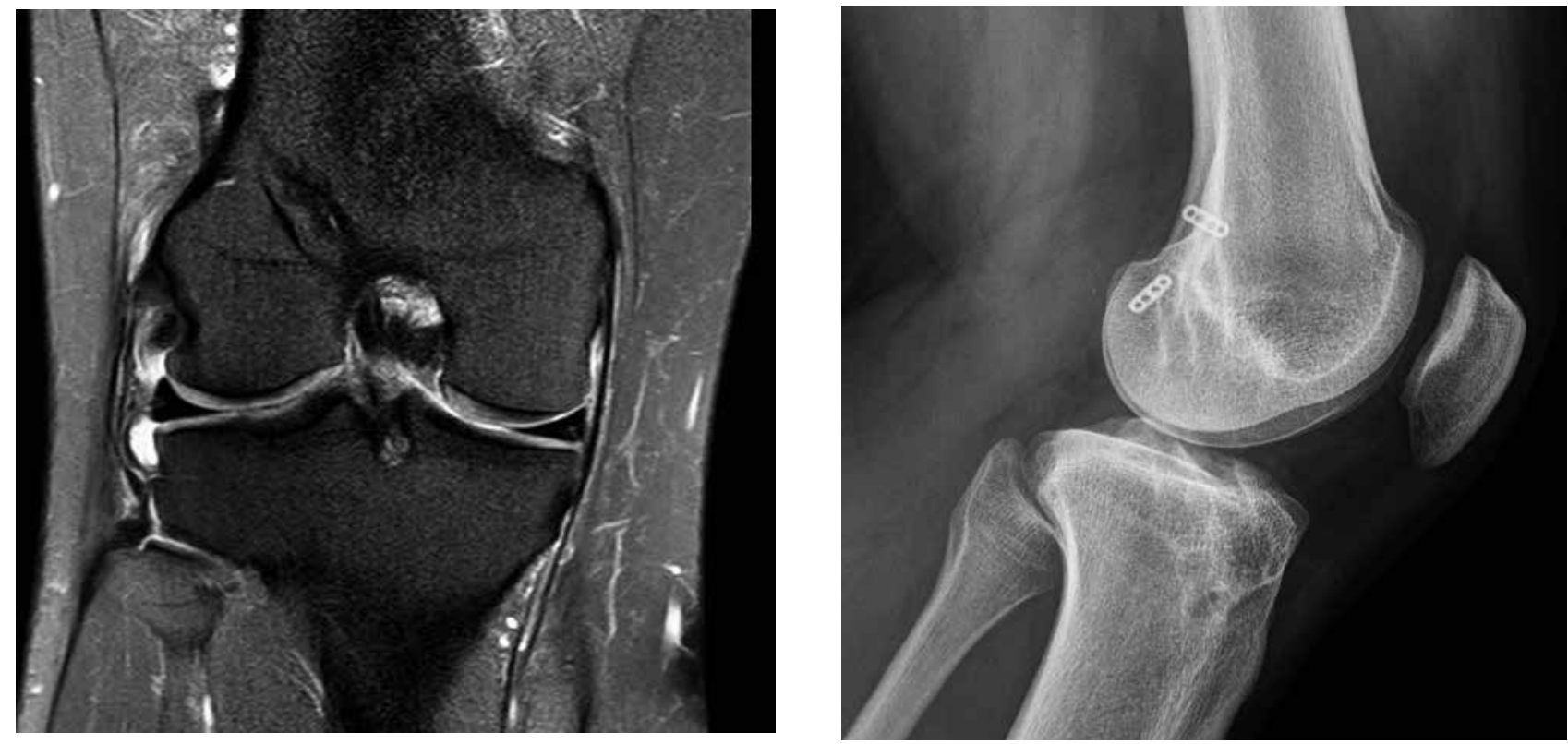

Figura 4. Radiografía frontal, lateral y cortes de resonancia magnética (2) coronal ponderada en DP-FS de rodilla derecha que muestra una reconstrucción "Doble banda", cuyo objetivo es lograr un injerto que cuente con bandas antero medial y postero lateral, de la misma manera que lo hace el ligamento cruzado anterior nativo.

\section{Conclusiones}

Las distintas técnicas quirúrgicas, en especial, las que buscan reconstrucción anatómica del LCA, producen variados hallazgos radiológicos, que no deben ser confundidos con patología. - El trabajo expone las técnicas más frecuentes y sus hallazgos normales, sirviendo de herramienta para el trabajo radiológico diario.

\section{Lectura recomendada}

1. Meyers A, Haims A, Menn K. Imaging of cruciate liga- ment repair and its complications. American Journal of radiology 2010; 194: 476-484.

2. Murasaki C, Wolf M, Araki D. Anatomic anterior cruciate ligament reconstruction: current concepts and future perspective. Cartilague 2014; 4: 27-37.

3. Grassi A, Bailey J, Signorelli C, Carbone G. Magnetic resonance imaging after anterior ligament reconstruction: a practical guide. Worl J orthop 2016; 7(10): 638-649.

4. Getgood A, Spalding T. The Evolution of Anatomic Anterior Cruciate Ligament Reconstruction. The Open Orthopaedics Journal, 2012; 6(2): 287-294. 